\title{
THE PROBABILITIES OF ABSOLUTE RUIN IN THE RENEWAL RISK MODEL WITH CONSTANT FORCE OF INTEREST
}

\author{
DIMITRIOS G. KONSTANTINIDES, ${ }^{*}$ University of the Aegean \\ KAI W. NG, ${ }^{* *}$ The University of Hong Kong \\ QIHE TANG, ${ }^{* * *}$ The University of Iowa
}

\begin{abstract}
In this paper we consider the probabilities of finite- and infinite-time absolute ruins in the renewal risk model with constant premium rate and constant force of interest. In the particular case of the compound Poisson model, explicit asymptotic expressions for the finite- and infinite-time absolute ruin probabilities are given. For the general renewal risk model, we present an asymptotic expression for the infinite-time absolute ruin probability. Conditional distributions of Poisson processes and probabilistic techniques regarding randomly weighted sums are employed in the course of this study.
\end{abstract}

Keywords: Absolute ruin; asymptotics; constant force of interest; convolution equivalence; heavy tail; renewal risk model

2010 Mathematics Subject Classification: Primary 91B30

Secondary 60G70; 60K05

\section{Introduction}

In this paper we address the probabilities of finite- and infinite-time absolute ruins in the renewal risk model with constant premium rate and constant force of interest. In this model, the claim sizes, $X_{k}, k=1,2, \ldots$, form a sequence of independent, identically distributed (i.i.d.), nonnegative random variables with generic random variable $X$ and common distribution $F=1-\bar{F}$. The interoccurrence times $\theta_{k}, k=1,2, \ldots$, form another sequence of i.i.d. positive random variables with generic random variable $\theta$. We assume that the sequences $\left\{\theta, \theta_{k}, k=1,2, \ldots\right\}$ and $\left\{X, X_{k}, k=1,2, \ldots\right\}$ are mutually independent. The occurrence times of the successive claims, $T_{n}=\sum_{k=1}^{n} \theta_{k}, n=1,2, \ldots$, constitute a renewal counting process

$$
N_{t}=\#\left\{T_{n} \leq t: n=1,2, \ldots\right\}, \quad t \geq 0 .
$$

Therefore, the compound renewal process, $C_{t}=\sum_{k=1}^{N_{t}} X_{k}$, represents aggregate claims up to time $t \geq 0$, with $C_{t}=0$ when $N_{t}=0$. Let $x \geq 0$ be the initial surplus of the insurance company, let $c>0$ be the constant premium rate, and let $\delta>0$ be the constant force of interest so that after time $t$ a capital $x$ becomes $x \mathrm{e}^{\delta t}$. Then the total surplus up to time $t$, denoted as

\footnotetext{
Received 21 September 2009.

* Postal address: Department of Statistics and Actuarial - Financial Mathematics, University of the Aegean, Karlovassi, GR-83 200 Samos, Greece. Email address: konstant@aegean.gr

** Postal address: Department of Statistics and Actuarial Science, The University of Hong Kong, Pokfulam Road, Hong Kong. Email address: kaing@hku.hk

*** Postal address: Department of Statistics and Actuarial Science, The University of Iowa, 241 Schaeffer Hall, Iowa City, IA 52242, USA. Email address: qtang@stat.uiowa.edu
} 
$W_{\delta}(t)$, is given by

$$
W_{\delta}(t)=x \mathrm{e}^{\delta t}+c \int_{0}^{t} \mathrm{e}^{\delta(t-y)} \mathrm{d} y-\int_{0}^{t} \mathrm{e}^{\delta(t-y)} \mathrm{d} C_{y}, \quad t \geq 0 .
$$

If the interoccurrence time $\theta$ is exponentially distributed with mean $1 / \lambda$, or, equivalently, $\left\{N_{t}, t \geq 0\right\}$ is a Poisson process with intensity $\lambda$, then the model above reduces to the compound Poisson risk model, also called the classical risk model.

In the actuarial literature, the probability of infinite-time ruin is defined to be the probability that the surplus falls below 0 . This probability has been extensively investigated in the compound Poisson model with constant force of interest; see, e.g. [1], [16], [19], [20], [23], [24], and [25].

As commented by Embrechts and Schmidli [10], the boundary zero here plays an unrealistic role. They used the alternative boundary $-c / \delta$. Whenever the surplus process hits this boundary, the company will not be able to repay its debts. Motivated by the work of [10], we define the probability of infinite-time absolute ruin as

$$
\psi(x, \infty)=\operatorname{Pr}\left(\inf _{t \geq 0} W_{\delta}(t)<-\frac{c}{\delta} \mid W_{\delta}(0)=x\right), \quad x \geq 0,
$$

and define the probability of finite-time absolute ruin as

$$
\psi(x, t)=\operatorname{Pr}\left(\inf _{0 \leq s \leq t} W_{\delta}(s)<-\frac{c}{\delta} \mid W_{\delta}(0)=x\right), \quad x \geq 0, t \geq 0 .
$$

Compared with the study on the ruin probabilities in the ordinary sense, the absolute ruin probabilities have received less attention than they deserve. In the compound Poisson model and for the general case with possibly different forces of interest for invested and borrowed money, using the technique of piecewise deterministic Markov processes and martingales, Embrechts and Schmidli [10, Theorem 1] showed an equality as an estimate for the infinite-time absolute ruin probability. This estimate involves a function that can be explicitly expressed in certain cases such as that of exponential claims, but it is not easy in general.

Absolute ruin was initially considered in [12] and further included in the book [13]. It is to be noted that in [8] martingale methods in the context of absolute ruin were applied and in [9] the influence of the force of interest on the negative surplus through several examples was described.

Recently, most of the works on absolute ruin have concentrated on the compound Poisson risk model. In [2] the Gerber-Shiu discounted penalty function was used to study the relation between the asymptotic expressions for the infinite-time absolute ruin probability and the ordinary infinite-time ruin probability. In [14] the compound Poisson risk model enriched with an independent Brownian motion was considered and their analysis was based on the jump diffusion model. There are calculations in three special examples with the corresponding numerical applications. In [30] an asymptotic formula for the infinite-time absolute ruin probability with different forces of interest for invested and borrowed money was established. In [29] the multilayer model with different premium rates on different layers of the surplus process and different forces of interest for invested and borrowed money in the framework of the compound Poisson risk model was examined.

This paper aims to provide asymptotic estimates for the absolute ruin probabilities defined in (1.2) and (1.3) as the initial surplus $x$ increases for the case where the claim sizes follow 
a distribution from the class $\delta(\gamma)$ for $\gamma \geq 0$. In doing so, we mainly apply some standard probabilistic arguments from [7], [15], [21], [26], [27], and [28].

The rest of this paper consists of three sections. As the starting point of the present research, we establish in Section 2 a proposition which presents a simple structure of the probability of absolute ruin as being the tail probability of a randomly weighted sum of nonnegative random variables, for both cases of finite and infinite time. In Section 3 three main results are shown, two providing explicit asymptotic estimates for the finite- and infinite-time absolute ruin probabilities in the compound Poisson model and one providing a general asymptotic estimate for the infinite-time absolute ruin probability in the general renewal model. In Section 4 we prove the main results after a series of lemmas.

\section{A treatment on the probabilities of absolute ruin}

At occurrence time $T_{n}=\sum_{k=1}^{n} \theta_{k}$, we observe the value $W_{\delta}\left(T_{n}\right)$ which represents the surplus immediately after paying the $n$th claim, $n=1,2, \ldots$ By virtue of (1.1) we can see that this sequence satisfies the recurrence equation

$$
W_{\delta}(0)=x, \quad W_{\delta}\left(T_{n}\right)=W_{\delta}\left(T_{n-1}\right) \mathrm{e}^{\delta \theta_{n}}+\frac{c}{\delta}\left(\mathrm{e}^{\delta \theta_{n}}-1\right)-X_{n}, \quad n=1,2, \ldots
$$

Consider another sequence

$$
V_{n}=W_{\delta}\left(T_{n}\right)+\frac{c}{\delta}, \quad n=0,1, \ldots
$$

It follows that

$$
V_{0}=x+\frac{c}{\delta}, \quad V_{n}=V_{n-1} \mathrm{e}^{\delta \theta_{n}}-X_{n}, \quad n=1,2, \ldots,
$$

and, hence, that

$$
V_{n}=\left(x+\frac{c}{\delta}\right) \prod_{k=1}^{n} \mathrm{e}^{\delta \theta_{k}}-\sum_{k=1}^{n} X_{k} \prod_{i=k+1}^{n} \mathrm{e}^{\delta \theta_{i}}, \quad n=1,2, \ldots
$$

Since absolute ruin can happen only at the time of a claim occurrence, we rewrite the infinitetime absolute ruin probability in (1.2) as

$$
\psi(x, \infty)=\operatorname{Pr}\left(\inf _{n \geq 1} W_{\delta}\left(T_{n}\right)<-\frac{c}{\delta} \mid W_{\delta}(0)=x\right) .
$$

With $Y_{k}=\mathrm{e}^{-\delta \theta_{k}}$ for $k=1,2, \ldots$, we further rewrite this probability as

$$
\begin{aligned}
\psi(x, \infty) & =\operatorname{Pr}\left(\inf _{n \geq 1} V_{n}<0 \mid V_{0}=x+\frac{c}{\delta}\right) \\
& =\operatorname{Pr}\left(\inf _{n \geq 1}\left(\left(x+\frac{c}{\delta}\right)-\sum_{k=1}^{n} X_{k} \prod_{i=1}^{k} Y_{i}\right)<0\right) \\
& =\operatorname{Pr}\left(\sum_{k=1}^{\infty} X_{k} \prod_{i=1}^{k} Y_{i}>x+\frac{c}{\delta}\right) .
\end{aligned}
$$


Relation (2.1) can be interpreted easily. Each term $X_{k} \prod_{i=1}^{k} Y_{i}$ is the discounted value of claim size $X_{k}$ according to the constant force of interest $\delta$. The sum $\sum_{k=1}^{\infty} X_{k} \prod_{i=1}^{k} Y_{i}$ denotes the total of all discounted future claims while the threshold for absolute ruin, $x+c / \delta$, denotes the initial surplus plus the total discounted premium.

Similarly, for the finite-time absolute ruin probability defined in (1.3), we have

$$
\begin{aligned}
\psi(x, t) & =\operatorname{Pr}\left(\inf _{1 \leq n \leq N_{t}} W_{\delta}\left(T_{n}\right)<-\frac{c}{\delta} \mid W_{\delta}(0)=x\right) \\
& =\operatorname{Pr}\left(\inf _{1 \leq n \leq N_{t}} V_{n}<0 \mid V_{0}=x+\frac{c}{\delta}\right) \\
& =\operatorname{Pr}\left(\sum_{k=1}^{N_{t}} X_{k} \prod_{i=1}^{k} Y_{i}>x+\frac{c}{\delta}\right) .
\end{aligned}
$$

We therefore record the following proposition.

Proposition 2.1. Consider the renewal risk model with constant force of interest $\delta>0$. The absolute ruin probabilities in (1.2) and (1.3) can be expressed as in (2.1) and (2.2), respectively.

Proposition 2.1, which forms the foundation of our study, rewrites the absolute ruin probabilities as the tail probabilities of randomly weighted sums. This gives rise to the opportunity of applying some techniques well developed in the study of randomly weighted sums. We also remark that relations (2.1) and (2.2) hold most generally, since in deriving them neither independence nor the i.i.d. assumption is used. However, in developing (2.1) and (2.2) our assumption of the same force of interest on invested and borrowed money is essential. Therefore, Proposition 2.1 cannot handle the case of varying force of interest.

\section{Main results}

Here and henceforth, all limit relationships are for $x \rightarrow \infty$ unless stated otherwise and the symbol ' $\sim$ ' means that the quotient of both sides tends to 1 . Clearly, for two positive functions $f(\cdot)$ and $g(\cdot)$, the relation $f(x) \sim g(x)$ amounts to the conjunction of the relations $\limsup f(x) / g(x) \leq 1$ and $\lim \inf f(x) / g(x) \geq 1$, which are denoted as $f(x) \lesssim g(x)$ and $f(x) \gtrsim g(x)$, respectively. For two distributions $F_{1}$ and $F_{2}$ on $[0, \infty)$, denote by $F_{1} * F_{2}$ their convolution; that is, for every $x \geq 0$,

$$
F_{1} * F_{2}(x)=\int_{0-}^{x} F_{1}(x-y) F_{2}(\mathrm{~d} y) .
$$

Furthermore, we write $F^{1 *}=F$ and $F^{n *}=F^{(n-1) *} * F$ for every $n=2,3, \ldots$

A distribution $F$ on $[0, \infty)$ is said to belong to the class $\delta(\gamma)$ for some $\gamma \geq 0$ if

$$
\lim _{x \rightarrow \infty} \frac{\bar{F}(x-y)}{\bar{F}(x)}=\mathrm{e}^{\gamma y}
$$

for every real number $y$ and the limit

$$
\lim _{x \rightarrow \infty} \frac{\overline{F^{2 *}}(x)}{\bar{F}(x)}=2 \int_{0-}^{\infty} \mathrm{e}^{\gamma y} F(\mathrm{~d} y)
$$


exists and is finite. A larger class, $\mathcal{L}(\gamma)$, is defined by relation (3.1) alone. For the wellknown subexponential class $\delta(0)$, when $\gamma=0$, the right-hand side of (3.2) becomes 2 . For two distributions, $F_{1} \in \mathcal{L}(\gamma)$ and $F_{2} \in \mathcal{L}(\gamma)$, satisfying $0<\liminf \overline{F_{1}}(x) / \overline{F_{2}}(x) \leq$ $\lim \sup \overline{F_{1}}(x) / \overline{F_{2}}(x)<\infty$, it is known that $F_{1} \in \delta(\gamma)$ if and only if $F_{2} \in \delta(\gamma)$; see, e.g. [17, pp. 133-134].

Since it was introduced in [3], [4], and [5], the class $\delta(\gamma)$ has been extensively investigated by many researchers and applied to various fields. This class is often used to model claim-size distributions; see, e.g. [11], [18], and [28].

Closely related is the class $\mathcal{R}_{-\infty}$ of distributions with rapidly varying tails, characterized by the relation

$$
\lim _{x \rightarrow \infty} \frac{\bar{F}(x y)}{\bar{F}(x)}=0, \quad y>1 .
$$

Clearly, if $F \in \mathcal{L}(\gamma)$ for some $\gamma>0$ then $F \in \mathcal{R}_{-\infty}$. A lot of distributions in the class $\delta(0)$ such as lognormal and Weibull distributions still belong to the class $\mathcal{R}_{-\infty}$.

For the compound Poisson model, the conditional joint distribution of the $n$ occurrence times given a fixed time of observation, $N_{t}=n$, lends easier evaluation of the weighted sum in (2.2). The interplay of this conditional distribution and the asymptotic approximation of convolution-equivalent tails entails the first main result in this paper.

Theorem 3.1. In the compound Poisson model with constant force of interest $\delta>0$, if $F \in$ $s(\gamma)$ for some $\gamma \geq 0$ then it holds that, for every $0<t<\infty$,

$$
\psi(x, t) \sim \lambda \exp \left\{\frac{\lambda}{\delta} \int_{\gamma \mathrm{e}^{-\delta t}}^{\gamma} \frac{\mathrm{E}^{s X}-1}{s} \mathrm{~d} s-\frac{\gamma c}{\delta}\right\} \int_{0}^{t} \bar{F}\left(x \mathrm{e}^{\delta s}\right) \mathrm{d} s .
$$

It is tempting to substitute $t=\infty$ into both sides of (3.3) to get an asymptotic expression for the infinite-time absolute ruin probability. But, in general, the repeated limits with respect to $x \rightarrow \infty$ and $t \rightarrow \infty$ of the ratio of both sides of (3.3) may depend on the order of the limits, yielding different results. It turns out, however, that this intuitive substitute result in the compound Poisson model is valid as a consequence of our next main result for the general renewal risk model.

Theorem 3.2. In the renewal risk model with constant force of interest $\delta>0$, if $F \in \delta(\gamma) \cap$ $\mathcal{R}_{-\infty}$ for some $\gamma \geq 0$ then

$$
\mathrm{Ee}^{\gamma S_{\infty}}<\infty, \text { where } S_{\infty}=\sum_{k=1}^{\infty} X_{k} \prod_{i=1}^{k} Y_{i}
$$

and

$$
\psi(x, \infty) \sim \mathrm{Ee}^{\gamma S_{\infty}} \operatorname{Pr}\left(X Y>x+\frac{c}{\delta}\right)
$$

where $Y=\mathrm{e}^{-\delta \theta}$ is the generic random variable of the sequence $\left\{Y_{k}=\mathrm{e}^{-\delta \theta_{k}}, k=1,2, \ldots\right\}$.

The expectation $\mathrm{Ee}^{\gamma S_{\infty}}$ appearing in relation (3.4) is generally unknown for $\gamma>0$. However, if we go back to the compound Poisson model then this quantity is explicitly available, as shown in the following last main result of the paper. 
Theorem 3.3. In the compound Poisson model with constant force of interest $\delta>0$, if $F \in$ $\delta(\gamma) \cap \mathcal{R}_{-\infty}$ for some $\gamma \geq 0$ then it holds that

$$
\psi(x, \infty) \sim \lambda \exp \left\{\frac{\lambda}{\delta} \int_{0}^{\gamma} \frac{\mathrm{Ee}^{s X}-1}{s} \mathrm{~d} s-\frac{\gamma c}{\delta}\right\} \int_{0}^{\infty} \bar{F}\left(x \mathrm{e}^{\delta s}\right) \mathrm{d} s .
$$

As remarked above, relation (3.5) corresponds to relation (3.3) with $t=\infty$.

\section{Proofs}

\subsection{Lemmas}

Lemma 4.1. Let $F, F_{1}$, and $F_{2}$ be three distributions on $[0, \infty)$ such that $F \in s(\gamma)$ and that the limit $l_{i}=\lim _{x \rightarrow \infty} \overline{F_{i}}(x) / \bar{F}(x)$ exists and is finite for $i=1,2$. Then

$$
\lim _{x \rightarrow \infty} \frac{\overline{F_{1} * F_{2}}(x)}{\bar{F}(x)}=l_{1} \int_{0-}^{\infty} \mathrm{e}^{\gamma y} F_{2}(\mathrm{~d} y)+l_{2} \int_{0-}^{\infty} \mathrm{e}^{\gamma y} F_{1}(\mathrm{~d} y) .
$$

Proof. See [21, Proposition 2].

Lemma 4.2. Let $F_{1}$ and $F_{2}$ be two distributions on $[0, \infty)$. If $F_{1} \in \delta(\gamma), F_{2} \in \mathcal{L}(\gamma)$, and $\overline{F_{2}}(x)=O\left(\overline{F_{1}}(x)\right)$, then $F_{1} * F_{2} \in \delta(\gamma)$ and

$$
\overline{F_{1} * F_{2}}(x) \sim \overline{F_{1}}(x) \int_{0-}^{\infty} \mathrm{e}^{\gamma y} F_{2}(\mathrm{~d} y)+\overline{F_{2}}(x) \int_{0-}^{\infty} \mathrm{e}^{\gamma y} F_{1}(\mathrm{~d} y) .
$$

Proof. See [6, Corollary 1].

Lemma 4.3. Let $\left\{N_{t}, t \geq 0\right\}$ be a Poisson process with occurrence times $T_{k}, k=1,2, \ldots$, and let $\left\{X_{k}, k=1,2, \ldots\right\}$ be a sequence of i.i.d. random variables independent of $\left\{N_{t}, t \geq 0\right\}$. Given $N_{t}=n$ for arbitrarily fixed $t>0$ and $n=1,2, \ldots$, the sum $\sum_{k=1}^{n} X_{k} \mathrm{e}^{-\delta T_{k}}$ is equal in distribution to the sum $\sum_{k=1}^{n} X_{k} \mathrm{e}^{-\delta t U_{k}}$, where the random vector $\left(U_{1}, \ldots, U_{n}\right)$ consists of i.i.d. random variables uniformly distributed on $(0,1)$ and is independent of the vector $\left(X_{1}, \ldots, X_{n}\right)$.

Proof. According to [22, Theorem 2.3.1], the conditional distribution of $\left(T_{1}, \ldots, T_{n}\right)$ given $N_{t}=n$ is the same as the distribution of the random vector $\left(t U_{(1, n)}, \ldots, t U_{(n, n)}\right)$, where $U_{(1, n)}, \ldots, U_{(n, n)}$ denote the order statistics of the $n$ random variables $U_{1}, \ldots, U_{n}$. Furthermore, since in the sum $\sum_{k=1}^{n} X_{k} \mathrm{e}^{-\delta t U_{(k, n)}}$ the vector $\left(X_{1}, \ldots, X_{n}\right)$ consists of i.i.d. random variables and is independent of $\left(U_{(1, n)}, \ldots, U_{(n, n)}\right)$, by rearrangement, this sum is equal in distribution to the sum $\sum_{k=1}^{n} X_{k} \mathrm{e}^{-\delta t U_{k}}$.

Lemma 4.4. For two independent nonnegative random variables $X$ and $Y$, if $X$ follows a distribution $F \in \delta(\gamma)$ and $Y$ follows a distribution with an upper endpoint

$$
1=\sup \{y: \operatorname{Pr}(Y \leq y)<1\},
$$

then the product $X Y$ still follows a distribution in the class $\&(\gamma)$.

Proof. See [26, Theorem 1.1].

Lemma 4.5. Let $F$ be a distribution on $[0, \infty)$. If $F \in \delta(\gamma)$ then

(i) it holds that, for each fixed $n=1,2, \ldots$,

$$
\overline{F^{n *}}(x) \sim n\left(\int_{0-}^{\infty} \mathrm{e}^{\gamma y} F(\mathrm{~d} y)\right)^{n-1} \bar{F}(x)
$$


(ii) for every $\varepsilon>0$, there exists some constant $C_{\varepsilon}>0$ such that the inequality

$$
\frac{\overline{F^{n *}}(x)}{\bar{F}(x)} \leq C_{\varepsilon}\left(\int_{0-}^{\infty} \mathrm{e}^{\gamma y} F(\mathrm{~d} y)+\varepsilon\right)^{n}
$$

holds for all $n=1,2, \ldots$ and all $x$.

Proof. See [4, p. 665].

\subsection{Proof of Theorem 3.1}

Starting with (2.2) and conditioning on $N_{t}$, we have

$$
\psi(x, t)=\sum_{n=1}^{\infty} \operatorname{Pr}\left(\sum_{k=1}^{n} X_{k} \mathrm{e}^{-\delta T_{k}}>x+\frac{c}{\delta} \mid N_{t}=n\right) \operatorname{Pr}\left(N_{t}=n\right) .
$$

By means of Lemma 4.3 we can have a sequence of i.i.d. random variables, $\left\{U, U_{k}, k=\right.$ $1,2, \ldots\}$, uniformly distributed on the interval $(0,1)$ and independent of $\left\{X, X_{k}, k=1,2, \ldots\right\}$, such that

$$
\psi(x, t)=\sum_{n=1}^{\infty} \operatorname{Pr}\left(\sum_{k=1}^{n} X_{k} \mathrm{e}^{-\delta t U_{k}}>x+\frac{c}{\delta}\right) \operatorname{Pr}\left(N_{t}=n\right) .
$$

By Lemma 4.4, the products $X_{k} \mathrm{e}^{-\delta t U_{k}}, k=1,2, \ldots$, are i.i.d. with common distribution belonging to the class $\delta(\gamma)$. Therefore, by Lemma $4.5(\mathrm{i})$, it holds that, for each $n=1,2, \ldots$,

$$
\operatorname{Pr}\left(\sum_{k=1}^{n} X_{k} \mathrm{e}^{-\delta t U_{k}}>x+\frac{c}{\delta}\right) \sim n \mathrm{e}^{-\gamma c / \delta}\left(\mathrm{E}^{\gamma X \mathrm{e}^{-\delta t U}}\right)^{n-1} \operatorname{Pr}\left(X \mathrm{e}^{-\delta t U}>x\right) .
$$

Applying the dominated convergence theorem justified by Lemma 4.5(ii), we obtain

$$
\begin{aligned}
\psi(x, t) & \sim \mathrm{e}^{-\gamma c / \delta} \sum_{n=1}^{\infty} n\left(\mathrm{Ee}^{\gamma X \mathrm{e}^{-\delta t U}}\right)^{n-1} \operatorname{Pr}\left(X \mathrm{e}^{-\delta t U}>x\right) \frac{(\lambda t)^{n}}{n !} \mathrm{e}^{-\lambda t} \\
& =\lambda t \mathrm{e}^{-\gamma c / \delta} \exp \left\{\lambda t\left(\mathrm{E} \mathrm{e}^{\gamma X \mathrm{e}^{-\delta t U}}-1\right)\right\} \operatorname{Pr}\left(X \mathrm{e}^{-\delta t U}>x\right) \\
& =\lambda \exp \left\{\frac{\lambda}{\delta} \int_{\gamma \mathrm{e}^{-\delta t}}^{\gamma} \frac{\mathrm{E}^{s X}-1}{s} \mathrm{~d} s-\frac{\gamma c}{\delta}\right\} \int_{0}^{t} \bar{F}\left(x \mathrm{e}^{\delta s}\right) \mathrm{d} s .
\end{aligned}
$$

This leads to (3.3).

\subsection{Proof of Theorem 3.2}

Our proof below is motivated by an idea in the proof of [15, Theorem 1]. Let $Z$ be a random variable with distribution $F$ and independent of $\left\{(X, Y),\left(X_{k}, Y_{k}\right), k=1,2, \ldots\right\}$, and denote the distribution of $Y=\mathrm{e}^{-\delta \theta}$ by $G$, which is supported on $(0,1)$. Then

$$
\begin{aligned}
\operatorname{Pr}((Z+X) Y>x) & =\int_{0}^{1} \operatorname{Pr}\left(Z+X>\frac{x}{y}\right) G(\mathrm{~d} y) \\
& \sim 2 \mathrm{Ee}^{\gamma X} \int_{0}^{1} \bar{F}\left(\frac{x}{y}\right) G(\mathrm{~d} y) \\
& =o(1) \bar{F}(x)
\end{aligned}
$$


where the second step is due to $F \in \delta(\gamma)$ and the last step is due to $F \in \mathcal{R}_{-\infty}$. Therefore, there is some $x_{0}>0$ large enough such that, for all $x>x_{0}$,

$$
\operatorname{Pr}((Z+X) Y>x) \leq \bar{F}(x)
$$

Construct a new conditional random variable $X^{*}=\left(Z \mid Z>x_{0}\right)$, whose distribution still belongs to the intersection $\delta(\gamma) \cap \mathcal{R}_{-\infty}$. Then, it is easy to see that

$$
\left(X^{*}+X\right) Y \stackrel{\mathrm{D}}{\leq} X^{*},
$$

or, equivalently, for all $x$,

$$
\operatorname{Pr}\left(\left(X^{*}+X\right) Y>x\right) \leq \operatorname{Pr}\left(Z>x \mid Z>x_{0}\right)
$$

where ' $\leq$ ' denotes 'stochastically not larger than'. Actually, when $x \leq x_{0}$, relation (4.3) is trivial as the right-hand side becomes equal to 1, while, when $x>x_{0}$, by (4.1),

$$
\begin{aligned}
\operatorname{Pr}\left(\left(X^{*}+X\right) Y>x\right) & =\frac{\operatorname{Pr}\left((Z+X) Y>x, Z>x_{0}\right)}{\operatorname{Pr}\left(Z>x_{0}\right)} \\
& \leq \frac{\operatorname{Pr}((Z+X) Y>x)}{\operatorname{Pr}\left(Z>x_{0}\right)} \\
& \leq \frac{\operatorname{Pr}(Z>x)}{\operatorname{Pr}\left(Z>x_{0}\right)} \\
& =\operatorname{Pr}\left(Z>x \mid Z>x_{0}\right) .
\end{aligned}
$$

Thus, relation (4.3) always holds. Relation (4.2) leads to

$$
\left(X^{*}+X_{1}\right) Y_{1} \stackrel{\mathrm{D}}{\leq} X^{*}, \quad\left(X^{*}+X_{2}\right) Y_{2} \stackrel{\mathrm{D}}{\leq} X^{*} .
$$

It follows that

$$
\left(\left(X^{*}+X_{2}\right) Y_{2}+X_{1}\right) Y_{1} \stackrel{\mathrm{D}}{\leq} X^{*} .
$$

Hence, $S_{T_{1}}=X_{1} Y_{1} \stackrel{\mathrm{D}}{\leq} X^{*}$ and $S_{T_{2}}=X_{1} Y_{1}+X_{2} Y_{2} Y_{1} \stackrel{\mathrm{D}}{\leq} X^{*}$. Repeating these iterations we obtain $S_{T_{n}} \stackrel{\text { D }}{\leq} X^{*}$ for every $n=1,2, \ldots$ Letting $n \rightarrow \infty$ yields

$$
S_{\infty} \stackrel{\mathrm{D}}{\leq} X^{*}
$$

which implies, as a by-product, that E $\mathrm{e}^{\gamma S_{\infty}}<\infty$. Let $\tilde{S}_{\infty}$ be a copy of $S_{\infty}$ independent of $\left\{\left(X_{k}, Y_{k}\right), k=1,2, \ldots\right\}$. Then, for every $n=1,2, \ldots$,

$$
S_{\infty} \stackrel{\mathrm{D}}{=} S_{T_{n}}+\tilde{S}_{\infty} \prod_{i=1}^{n} Y_{i},
$$

where ' $\stackrel{\mathrm{D}}{=}$ ' denotes equality in distribution. Therefore,

$$
S_{\infty} \stackrel{\mathrm{D}}{\leq} S_{T_{n}}+X^{*} \prod_{i=1}^{n} Y_{i} .
$$


From this we obtain, for each $n=2,3, \ldots$,

$$
\begin{aligned}
\operatorname{Pr}\left(S_{\infty}>x\right) & \leq \operatorname{Pr}\left(S_{T_{n}}+X^{*} \prod_{i=1}^{n} Y_{i}>x\right) \\
& =\int_{0}^{1} \operatorname{Pr}\left(X_{1}+\sum_{k=2}^{n} X_{k} \prod_{i=2}^{k} Y_{i}+X^{*} \prod_{i=2}^{n} Y_{i}>\frac{x}{y}\right) G(\mathrm{~d} y) .
\end{aligned}
$$

By Lemma 4.2,

$$
\begin{aligned}
\operatorname{Pr}\left(\sum_{k=2}^{n} X_{k} \prod_{i=2}^{k} Y_{i}+X^{*} \prod_{i=2}^{n} Y_{i}>x\right) \\
\quad \leq \operatorname{Pr}\left(\left(\sum_{k=2}^{n} X_{k}+X^{*}\right) Y_{2}>x\right) \\
\quad=\int_{0}^{1} \operatorname{Pr}\left(\sum_{k=2}^{n} X_{k}+X^{*}>\frac{x}{y}\right) G(\mathrm{~d} y) \\
\quad \sim\left(\mathrm{Ee}^{\gamma X^{*}}(n-1)\left(\mathrm{E}^{\gamma X}\right)^{n-2}+\frac{\left(\mathrm{E} \mathrm{e}^{\gamma X}\right)^{n-1}}{\bar{F}\left(x_{0}\right)}\right) \int_{0}^{1} \operatorname{Pr}\left(X>\frac{x}{y}\right) G(\mathrm{~d} y) \\
\quad=o(1) \bar{F}(x),
\end{aligned}
$$

where the last step is due to $F \in \mathcal{R}_{-\infty}$. Now we apply Lemma 4.1 to continue the derivation of (4.4) to find that

$$
\begin{aligned}
\operatorname{Pr}\left(S_{\infty}>x\right) & \lesssim \int_{0}^{1} \operatorname{E} \exp \left\{\gamma\left(\sum_{k=2}^{n} X_{k} \prod_{i=2}^{k} Y_{i}+X^{*} \prod_{i=2}^{n} Y_{i}\right)\right\} \operatorname{Pr}\left(X_{1}>\frac{x}{y}\right) G(\mathrm{~d} y) \\
& =\operatorname{E} \exp \left\{\gamma\left(\sum_{k=2}^{n} X_{k} \prod_{i=2}^{k} Y_{i}+X^{*} \prod_{i=2}^{n} Y_{i}\right)\right\} \operatorname{Pr}(X Y>x),
\end{aligned}
$$

or, equivalently,

$$
\limsup _{x \rightarrow \infty} \frac{\operatorname{Pr}\left(S_{\infty}>x\right)}{\operatorname{Pr}(X Y>x)} \leq \operatorname{Eexp}\left\{\gamma\left(\sum_{k=2}^{n} X_{k} \prod_{i=2}^{k} Y_{i}+X^{*} \prod_{i=2}^{n} Y_{i}\right)\right\}
$$

Clearly, $\sum_{k=2}^{n} X_{k} \prod_{i=2}^{k} Y_{i}+X^{*} \prod_{i=2}^{n} Y_{i}$ converges to $S_{\infty}$ in distribution as $n \rightarrow \infty$. Therefore, by the dominated convergence theorem, the expectation on the right-hand side above converges to $\mathrm{E}^{\gamma S_{\infty}}$ as $n \rightarrow \infty$. This establishes the asymptotic upper bound as

$$
\limsup _{x \rightarrow \infty} \frac{\operatorname{Pr}\left(S_{\infty}>x\right)}{\operatorname{Pr}(X Y>x)} \leq \mathrm{Ee}^{\gamma S_{\infty}}
$$


It is easier to construct the corresponding asymptotic lower bound. Similarly as above,

$$
\begin{aligned}
\operatorname{Pr}\left(S_{\infty}>x\right) & \geq \operatorname{Pr}\left(S_{T_{n}}>x\right) \\
& =\int_{0}^{1} \operatorname{Pr}\left(X_{1}+\sum_{k=2}^{n} X_{k} \prod_{i=2}^{k} Y_{i}>\frac{x}{y}\right) G(\mathrm{~d} y) \\
& \sim \operatorname{E} \exp \left\{\gamma\left(\sum_{k=2}^{n} X_{k} \prod_{i=2}^{k} Y_{i}\right)\right\} \operatorname{Pr}(X Y>x),
\end{aligned}
$$

or, equivalently,

$$
\liminf _{x \rightarrow \infty} \frac{\operatorname{Pr}\left(S_{\infty}>x\right)}{\operatorname{Pr}(X Y>x)} \geq \operatorname{E} \exp \left\{\gamma\left(\sum_{k=2}^{n} X_{k} \prod_{i=2}^{k} Y_{i}\right)\right\} .
$$

Clearly, $\sum_{k=2}^{n} X_{k} \prod_{i=2}^{k} Y_{i}$ converges to $S_{\infty}$ in distribution as $n \rightarrow \infty$. Therefore, the expectation on the right-hand side above converges to $\mathrm{E}^{\gamma S_{\infty}}$ as $n \rightarrow \infty$ too. We have

$$
\liminf _{x \rightarrow \infty} \frac{\operatorname{Pr}\left(S_{\infty}>x\right)}{\operatorname{Pr}(X Y>x)} \geq \mathrm{Ee}^{\gamma S_{\infty}} .
$$

This completes the proof of Theorem 3.2.

\subsection{Proof of Theorem 3.3}

We first calculate the factor $\mathrm{E}^{\gamma S_{\infty}}$ of (3.4) in the framework of the compound Poisson model. As in the proof of Theorem 3.1, applying Lemma 4.3 to

$$
S_{t}=\sum_{k=1}^{N_{t}} X_{k} \prod_{i=1}^{k} Y_{i}=\sum_{k=1}^{N_{t}} X_{k} \mathrm{e}^{-\delta T_{k}}
$$

we have a sequence of i.i.d. random variables, $\left\{U, U_{k}, k=1,2, \ldots\right\}$, uniformly distributed on the interval $(0,1)$ and independent of $\left\{X, X_{k}, k=1,2, \ldots\right\}$, such that

$$
\begin{aligned}
\mathrm{Ee}^{\gamma S_{t}} & =\sum_{n=0}^{\infty} \mathrm{E}\left(\exp \left\{\gamma \sum_{k=1}^{N_{t}} X_{k} \mathrm{e}^{-\delta T_{k}}\right\} \mid N_{t}=n\right) \operatorname{Pr}\left(N_{t}=n\right) \\
& =\sum_{n=0}^{\infty} \mathrm{E}\left(\exp \left\{\gamma \sum_{k=1}^{n} X_{k} \mathrm{e}^{-\delta t U_{k}}\right\}\right) \operatorname{Pr}\left(N_{t}=n\right) \\
& =\sum_{n=0}^{\infty}\left(\mathrm{E} \mathrm{e}^{\gamma X \mathrm{e}^{-\delta t U}}\right)^{n} \frac{(\lambda t)^{n}}{n !} \mathrm{e}^{-\lambda t} \\
& =\exp \left\{\lambda t\left(\mathrm{E} \mathrm{e}^{\gamma X \mathrm{e}^{-\delta t U}}-1\right)\right\}
\end{aligned}
$$

It follows that

$$
\begin{aligned}
\mathrm{Ee}^{\gamma S_{\infty}} & =\lim _{t \rightarrow \infty} \mathrm{E}^{\gamma S_{t}} \\
& =\lim _{t \rightarrow \infty} \exp \left\{\lambda t\left(\mathrm{E} \mathrm{e}^{\gamma X \mathrm{e}^{-\delta t U}}-1\right) t\right\} \\
& =\exp \left\{\lim _{t \rightarrow \infty} \lambda \int_{0}^{t}\left(\mathrm{E}^{\gamma X \mathrm{e}^{-\delta s}}-1\right) \mathrm{d} s\right\} \\
& =\exp \left\{\frac{\lambda}{\delta} \int_{0}^{\gamma} \frac{\mathrm{E}^{s X}-1}{s} \mathrm{~d} s\right\} .
\end{aligned}
$$


We then calculate the probability $\operatorname{Pr}(X Y>x+c / \delta)$ in (3.4). Since $F \in \mathcal{R}_{-\infty}$, by [26, Lemma 3.1(i)], it holds that, for every $\varepsilon>0$,

$$
\operatorname{Pr}(X Y>x) \sim \int_{0}^{\varepsilon} \bar{F}\left(x \mathrm{e}^{\delta s}\right) \lambda \mathrm{e}^{-\lambda s} \mathrm{~d} s
$$

and that

$$
\int_{0}^{\varepsilon} \bar{F}\left(x \mathrm{e}^{\delta s}\right) \mathrm{d} s \sim \int_{0}^{\infty} \bar{F}\left(x \mathrm{e}^{\delta s}\right) \mathrm{d} s .
$$

By the arbitrariness of $\varepsilon>0$, it follows that

$$
\operatorname{Pr}(X Y>x) \sim \lambda \int_{0}^{\infty} \bar{F}\left(x \mathrm{e}^{\delta s}\right) \mathrm{d} s .
$$

Note that the distribution of $X Y$ still belongs to the class $\delta(\gamma)$ according to Lemma 4.4. Hence,

$$
\operatorname{Pr}\left(X Y>x+\frac{c}{\delta}\right) \sim \mathrm{e}^{-\gamma c / \delta} \operatorname{Pr}(X Y>x) \sim \lambda \mathrm{e}^{-\gamma c / \delta} \int_{0}^{\infty} \bar{F}\left(x \mathrm{e}^{\delta s}\right) \mathrm{d} s .
$$

Substituting (4.5) and (4.6) into (3.4) yields relation (3.5).

\section{Acknowledgement}

The authors would like to thank the anonymous referee for his/her careful reading of an earlier version of this paper.

\section{References}

[1] Asmussen, S. (1998). Subexponential asymptotics for stochastic processes: extremal behavior, stationary distributions and first passage probabilities. Ann. Appl. Prob. 8, 354-374.

[2] CaI, J. (2007). On the time value of absolute ruin with debit interest. Adv. Appl. Prob. 39, 343-359.

[3] Chistyakov, V. P. (1964). A theorem on sums of independent positive random variables and its applications to branching random processes. Theory Prob. Appl. 9, 640-648.

[4] Chover, J., Ney, P. And Wainger, S. (1973). Degeneracy properties of subcritical branching processes. Ann. Prob. 1, 663-673.

[5] Chover, J., Ney, P. And Wainger, S. (1973). Functions of probability measures. J. Analyse Math. 26, $255-302$.

[6] Cline, D. B. H. (1986). Convolution tails, product tails and domains of attraction. Prob. Theory Relat. Fields 72, 529-557.

[7] Cline, D. B. H. and Samorodnitsky, G. (1994). Subexponentiality of the product of independent random variables. Stoch. Process. Appl. 49, 75-98.

[8] Dassios, A. and Embrechts, P. (1989). Martingales and insurance risk. Commun. Statist. Stoch. Models 5, 181-217.

[9] Dickson, D. C. M. And Egídio dos ReIs, A. D. (1997). The effect of interest on negative surplus. Insurance Math. Econom. 21, 1-16.

[10] Embrechts, P. and Schmidli, H. (1994). Ruin estimation for a general insurance risk model. Adv. Appl. Prob. 26, 404-422.

[11] Embrechts, P. and Veraverbeke, N. (1982). Estimates for the probability of ruin with special emphasis on the possibility of large claims. Insurance Math. Econom. 1, 55-72.

[12] Gerber, H. U. (1971). Der Einfluss von Zins auf die Ruinwahrscheinlichkeit. Bull. Swiss Assoc. Actuaries 71, 63-70.

[13] Gerber, H. U. (1979). An Introduction to Mathematical Risk Theory (S. S. Huebner Foundation Monogr. Ser. 8). University of Pennsylvania, Philadelphia, PA.

[14] Gerber, H. U. and Yang, H. (2007). Absolute ruin probabilities in a jump diffusion risk model with investment. N. Amer. Actuarial J. 11, 159-169.

[15] Grey, D. R. (1994). Regular variation in the tail behaviour of solutions of random difference equations. Ann. Appl. Prob. 4, 169-183. 
[16] Kalashnikov, V. and Konstantinides, D. (2000). Ruin under interest force and subexponential claims: a simple treatment. Insurance Math. Econom. 27, 145-149.

[17] KlüPPElberg, C. (1988). Subexponential distributions and integrated tails. J. Appl. Prob. 25, 132-141.

[18] KlüPPELBERG, C. (1989). Estimation of ruin probabilities by means of hazard rates. Insurance Math. Econom. 8, 279-285.

[19] KlüPPElberg, C. ANd Stadtmüller, U. (1998). Ruin probabilities in the presence of heavy-tails and interest rates. Scand. Actuarial J. 1998, 49-58.

[20] Konstantinides, D., Tang, Q. And Tsitsiashvili, G. (2002). Estimates for the ruin probability in the classical risk model with constant interest force in the presence of heavy tails. Insurance Math. Econom. 31, 447-460.

[21] Rogozin, B. A. And Sgibnev, M. S. (1999). Banach algebras of measures on the line with given asymptotics of distributions at infinity. Siberian Math. J. 40, 565-576.

[22] Ross, S. M. (1983). Stochastic Processes. John Wiley, New York.

[23] Sundt, B. And Teugels, J. L. (1995). Ruin estimates under interest force. Insurance Math. Econom. 16, 7-22.

[24] Sundt, B. And Teugels, J. L. (1997). The adjustment function in ruin estimates under interest force. Insurance Math. Econom. 19, 85-94.

[25] TANG, Q. (2005). The finite-time ruin probability of the compound Poisson model with constant interest force. J. Appl. Prob. 42, 608-619.

[26] TANG, Q. (2006). On convolution equivalence with applications. Bernoulli 12, 535-549.

[27] Tang, Q. and Tsitsiashvili, G. (2003). Precise estimates for the ruin probability in finite horizon in a discretetime model with heavy-tailed insurance and financial risks. Stoch. Process. Appl. 108, 299-325.

[28] Tang, Q. And Tsitsiashvili, G. (2004). Finite- and infinite-time ruin probabilities in the presence of stochastic returns on investments. Adv. Appl. Prob. 36, 1278-1299.

[29] YANG, H., Zhang, Z. AND LAN, C. (2004). On the time value of absolute ruin for a multi-layer compound Poisson model under interest force. Statist. Prob. Lett. 78, 1835-1845.

[30] ZHU, J. AND YANG, H. (2008). Estimates for the absolute ruin probability in the compound Poisson risk model with credit and debit interest. J. Appl. Prob. 45, 818-830. 